\title{
Underground Study of Big Bang Nucleosynthesis in the Preci- sion Era of Cosmology
}

\author{
Carlo Gustavino ${ }^{1, a}$ \\ ${ }^{1}$ INFN, Sezione di Roma, Piazzale A. Moro 2, 00185 Roma, Italy
}

\begin{abstract}
Big Bang Nucleosinthesis (BBN) theory provides definite predictions for the abundance of light elements produced in the early universe, as far as the knowledge of the relevant nuclear processes of the BBN chain is accurate. At BBN energies $\left(30 \lesssim E_{c m} \lesssim 300 \mathrm{MeV}\right)$ the cross section of many BBN processes is very low because of the Coulomb repulsion between the interacting nuclei. For this reason it is convenient to perform the measurements deep underground. Presently the world's only facility operating underground is LUNA (Laboratory for Undergound Nuclear astrophysics) at LNGS ("Laboratorio Nazionale del Gran Sasso", Italy). In this presentation the BBN measurements of LUNA are briefly reviewed and discussed. It will be shown that the ongoing study of the $D(p, \gamma)^{3} \mathrm{He}$ reaction is of primary importance to derive the baryon density of universe $\Omega_{b}$ with high accuracy. Moreover, this study allows to constrain the existence of the so called "dark radiation", composed by undiscovered relativistic species permeating the universe, such as sterile neutrinos.
\end{abstract}

\section{Big Bang Nucleosynthesis}

Big Bang Nucleosynthesis (BBN) occurs in the first minutes of cosmic time, as result of the competition between the expansion of the Universe and the nuclear reaction rates. The expansion rate of the universe is governed by the Freidmann equation:

$$
H^{2}=\frac{8 \pi}{3} G \rho
$$

Were $H$ is the Hubble parameter, $G$ is the Newton's gravitational constant and $\rho$ is the energy density which, in the early Universe, is dominated by the "radiation", i.e. the contributions from massless or extremely relativistic particles. The radiation density is often expressed as follows:

$$
\rho=\rho_{\gamma}\left(1+\frac{7}{8}\left(\frac{4}{11}\right)^{4 / 3} N_{e f f}\right)
$$

In this formula $\rho_{\gamma}$ is the photon density and $N_{e f f}$ is the contribution of other relativistic species. Using this formula $N_{e f f}=3.046$ if only the three known neutrino families are considered.

Constraints on cosmology and particle physics can be obtained by comparing the result of direct

\footnotetext{
a e-mail: carlo.gustavino@roma1.infn.it
} 
observations of light isotopes $\left({ }^{2} \mathrm{H},{ }^{3} \mathrm{He},{ }^{4} \mathrm{He},{ }^{6} \mathrm{Li},{ }^{7} \mathrm{Li}\right)$ in primordial astrophysical sites with BBN calculated abundances. Therefore, are very important both the accuracy of astronomical observations and the precise determination of nuclear cross sections of BBN leading processes.

\section{Nuclear Astrophysics}

At energies of interest in astroparticle physics $(0.01 \div 1 \mathrm{MeV})$ the cross-section $\sigma(E)$ drops almost exponentially with decreasing energy $E$, due to the repulsion of charged nuclei. For this reason, in nuclear astrophysics the nuclear cross section $\sigma(E)$ is often factorized as follows:

$$
\sigma(E)=\frac{S(E) e^{-2 \pi \eta}}{E}
$$

In this formula, the exponential term takes into account the Coulomb barrier, while the astrophysical factor $S(E)$ contains all the nuclear effects. The Sommerfeld parameter $\eta$ is given by $2 \pi \eta=31.29 Z_{1} Z_{2}(\mu / E)^{1 / 2} . Z_{1}$ and $Z_{2}$ are the nuclear charges of the interacting nuclei. $\mu$ is their reduced mass (in units of a.m.u.), and $E$ is the center of mass energy (in units of $\mathrm{keV}$ ). Due to the low reaction yield, direct measurements at low energy are severely hampered by the background induced by cosmic rays. For this reason the LUNA collaboration carries out its measurements with the world's only underground accelerator facility, operating at the "Laboratori Nazionali del Gran Sasso" (LNGS). In fact, the ultra-low background at LNGS makes possible to study the nuclear reactions well below the Coulomb barrier.

The BBN production of ${ }^{7} \mathrm{Li}$ is dominated by the ${ }^{3} \mathrm{He}(\alpha, \gamma){ }^{7} \mathrm{Be}$ reaction, with subsequent decay of radioactive ${ }^{7} \mathrm{Be}$ to ${ }^{7} \mathrm{Li}$. The ${ }^{3} \mathrm{He}(\alpha, \gamma){ }^{7} \mathrm{Be}$ reaction was studied at LUNA using two different experimental techniques: by detecting the promptly emitted $\gamma$-rays from the reaction and by measuring the ${ }^{7} \mathrm{Be}$ activity created in the experiment. The setup consisted of a windowless ${ }^{3} \mathrm{He}$ gas target, a ${ }^{4} \mathrm{He}^{+}$ beam and a high purity germanium detector (HPGe) to measure the prompt $\gamma$ 's yield. The ${ }^{7} \mathrm{Be}$ activity of the beam stopper were also measured with a HPGe detector installed underground. Figure 1 shows the LUNA result and literature data [1]. Note that only the LUNA data are well inside the BBN energy region. The LUNA result exacerbates the "lithium problem", excluding a nuclear solution to solve the tension between theory and observations.

Standard BBN production of ${ }^{6} \mathrm{Li}$ is dominated by just one nuclear reaction, ${ }^{2} \mathrm{H}(\alpha, \gamma){ }^{6} \mathrm{Li}$. Before LUNA no direct measurements were performed inside the BBN energy region, making the BBN calculation concerning ${ }^{6} \mathrm{Li}$ very uncertain. The ${ }^{2} \mathrm{H}(\alpha, \gamma){ }^{6} \mathrm{Li}$ S-factor has been recently measured at low energy by LUNA, with an $\alpha$ beam and a windowless deuterium gas target. The main problems for this experiment were the very low cross section (about $60 \mathrm{pb}$ at $E_{c m}=133 \mathrm{keV}$ ) and the existence of a significant beam induced background. Figure 1 shows the LUNA result together with previous direct measurements [1,2] and theoretical calculations [3]. The direct LUNA measurement excludes a nuclear solution to explain the debated overabundance of this isotope in metal poor stars.

The deuterium abundance is very important because it is determined from direct observations at the level of $1 \%$ [4] and it is very sensitive to $\Omega_{b}$ and to $N_{\text {eff }}$. The primordial abundance of deuterium depends on the four reactions shown in table 1. Its theoretical error is twice with respect to the uncertainty obtained by direct observations, and it is mainly due to the uncertainty of ${ }^{2} \mathrm{H}(\mathrm{p}, \gamma)^{3} \mathrm{He}$ cross section data [8]. The uncertainty due to this reaction is even larger if ab initio prediction is taken into account. Figure 1 shows the data of the ${ }^{2} H(p, \gamma)^{3} H e$ reaction in literature. Note that only a single dataset of $S_{12}$ is currently available in the relevant BBN energy range, in which the authors state systematic uncertainty of $9 \%$ [6]. However the fit of experimental data is about $20 \%$ lower than the theoretical calculation [7]. In this plot the LUNA data obtained with the previous 50 

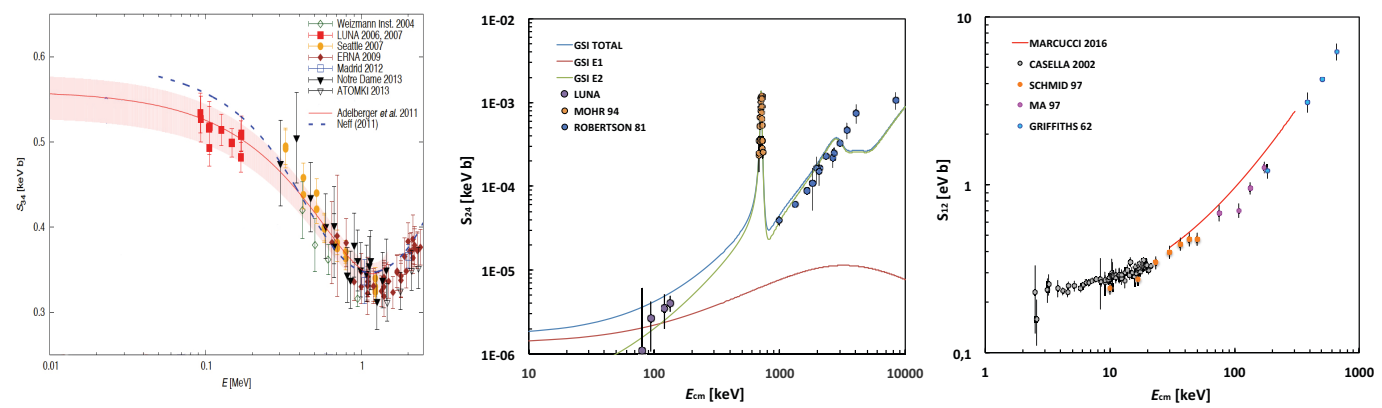

Figure 1. Left: astrophysical S-factor data of the ${ }^{3} \mathrm{He}(\alpha, \gamma)^{7}$ Be reaction as a function of the center-of-mass energy. A theoretical curve and the ab initio prediction are also shown. Center: astrophysical S-factor data of the ${ }^{2} H(\alpha, \gamma)^{6}$ Li reaction as a function of the center-of-mass energy. The LUNA data are shown with all the previous direct measurements. The continuous lines show the theoretical E1, E2, and total $S_{24}$ factors describing recent Coulomb dissociation data. Right: $S$-factor data for the reaction ${ }^{2} \mathrm{H}(p, \gamma)^{3} \mathrm{He}$. the red solid curve shows the prediction of recent ab initio theoretical calculation. See [1] and references therein.

Table 1. List of the leading reactions and corresponding rate symbols controlling the deuterium abundance after BBN. The last column shows the error on the ratio D/H coming from the uncertainty in the cross section of each reaction, for a fixed baryon density $\Omega_{b} h^{2}=0.02207$ [8].

\begin{tabular}{|l|c|c|}
\hline Reaction & Rate Symbol & $\sigma_{D / H} \cdot 10^{5}$ \\
\hline \hline$p(n, \gamma)^{2} H$ & $R_{1}$ & \pm 0.002 \\
$d(p, \gamma)^{3} H e$ & $R_{2}$ & \pm 0.062 \\
$d(d, n)^{3} H e$ & $R_{3}$ & \pm 0.020 \\
$d(d, p)^{3} H$ & $R_{4}$ & \pm 0.0013 \\
\hline
\end{tabular}

$\mathrm{kV}$ pilot accelerator are also shown. The present LUNA $400 \mathrm{kV}$ facility make possible to extend the measurements up to $E_{c m}=266 \mathrm{keV}$, i.e. well inside the BBN energy range. Figure 2a) shows the scheme of the setup where a barrel BGO detector is implemented. The ongoing measurements are performed with the proton beam produced by the $400 \mathrm{kV}$ accelerator, a windowless deuterium gas target and a $4 \pi$ BGO crystal to detect prompt $\gamma$ s [5]. The study with the BGO detector will be accomplished by using a different layout in which a Germanium detector is faced to the gas target in a close geometry, as shown in figure $2 b$ ). With this configuration can be inferred the angular distribution of photons emitted by the ${ }^{2} H(p, \gamma)^{3} H e$ reaction, exploiting the doppler effect affecting the energy of $\gamma$ 's produced along the beam line. Figure 3 shows the result of a preliminary measurement measurement performed with the HPGe detector. The energy distribution of emitted photons is well in agreement with ab initio calculation (see figure caption).

\section{Conclusions}

The lack of data of the ${ }^{2} \mathrm{H}(\mathrm{p}, \gamma)^{3} \mathrm{He}$ reaction is presently the main obstacle to improve the accuracy of $\Omega_{b}$ and $N_{e f f}$ values. The study of the ${ }^{2} H(p, \gamma)^{3} H e$ reaction in the BBN energy range is in progress 


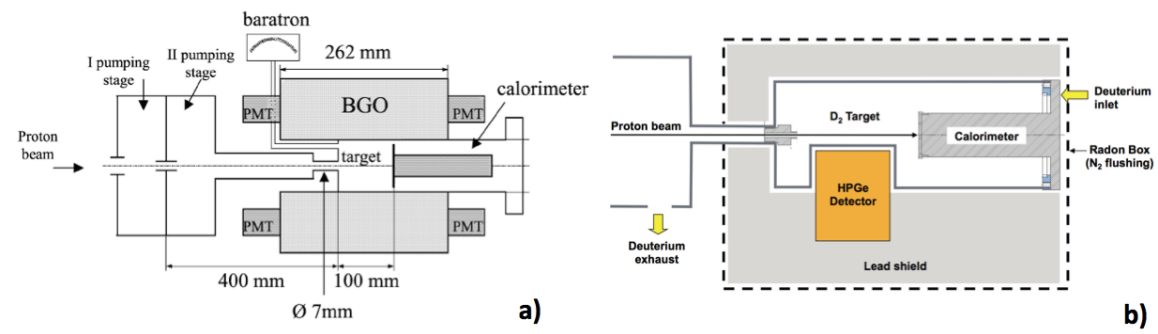

Figure 2. a): Scheme of gas target setup and BGO detector. b): Scheme of gas target setup and HPGe detector.

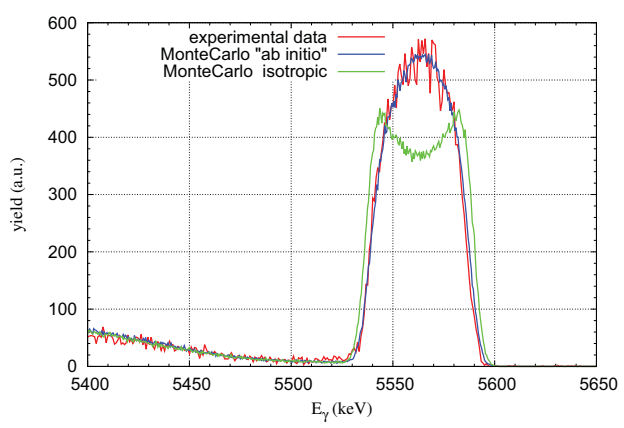

Figure 3. Simulated spectra of the ${ }^{2} H(p, \gamma)^{3} H e$ reaction, assuming isotropic (green) and ab initio (blue) angular distribution at $E_{c m}=112.5 \mathrm{keV}$. The experimental data (red) are also shown. Data have been normalized to remark the close agreement with the ab initio angular distribution.

with the LUNA400 facility at the underground Gran Sasso laboratory. The goal is to reach an accuracy at the $3 \%$ level, considerably better than the $9 \%$ uncertainty estimated in [6] and much lower than the $20 \%$ difference between data and nuclear calculations.

\section{References}

[1] C. Gustavino et al, Eur. Phys. J. A (2016) 52, 74 (2016).

[2] M. Anders et al, PRL 113, 042501 (2014).

[3] F. Hammache et al, Phys. Rev. C 82, 065803 (2010).

[4] R. Cooke et al, ApJ. 781, 31, (2014).

[5] C. Casella et al, Nuclear Physics A 706 20316. (2002).

[6] L. Ma et al, Phys. Rev. C 55, 588 (1997).

[7] L.E. Marcucci et al, PRL 116, 102501 (2016).

[8] E. Di Valentino et al, Phys. Rev. D 90, 023543 (2014). 